\title{
PROFESORES QUE ESTUDIAN PROFESORES. REFLEXIONES TEÓRICO-METODOLÓGICAS SOBRE LOS PROCESOS DE INVESTIGACIÓN EN Y SOBRE LA EDUCACIÓN FÍSICA
}

TEACHERS STUDYING TEACHERS. THEORETICAL AND METHODOLOGICAL REFLECTIONS ON RESEARCH PROCESSES IN AND ABOUT PHYSICAL EDUCATION

PROFESSORES QUE ESTUDAM PROFESSORES. REFLEXÕES TEÓRICOMETODOLÓGICAS SOBRE OS PROCESSOS DE PESQUISA EM E NA EDUCAÇÃO FÍSICA

Resumen

\begin{abstract}
En el presente ensayo se busca promover un conjunto de reflexiones teórico-metodológicas sobre las prácticas de investigación en la Educación Física. A partir del análisis de diferentes situaciones de trabajo de campo, producidas en dos investigaciones, se busca problematizar el proceso de producción de los datos y las interpretaciones. Con base en ello, es de interés poner en consideración del lector los diferentes procesos de reflexión y crítica que realizaron los diferentes sujetos que participaron de la situación de pesquisa —investigador e investigados-, nodales en la producción de los datos. Ello posibilitó visibilizar cómo las prácticas de investigación y sus resultados forman parte también de las disputas disciplinares.
\end{abstract}

Palabras clave: investigación; Educación Física; metodología; teoría

Abstract

This essay seeks to promote a group of theoretical-methodological reflections on research practices in Physical Education. Based on the analysis of different field work situations produced in two investigations, we seek to problematize the process of data production and interpretation. Based on this, it is interesting to put in consideration of the reader the different processes of reflection and criticism that the different subjects that participated in the research situation - researcher and researched-, nodal in the production of the data. This made it possible to make visible how investigative practices and their results also form part of disciplinary disputes.

Keywords: research; physical Education; methodology; theory

1 Universidad Nacional de La Plata, Argentina. Correo electrónico: levoratti@gmail.com 


\section{Resumo}

O presente ensaio busca promover um conjunto de reflexões teórico-metodológicas sobre as práticas de pesquisa em Educação Física. Em particular, é interessante colocar na consideração do leitor os diferentes processos de reflexão e crítica realizados pelos diferentes sujeitos que participaram da situação de pesquisa -pesquisador e investigado-, que foram centrais na produção dos dados. Isso permitiu tornar visível como as práticas de pesquisa e seus resultados também fazem parte das disputas disciplinares.

Palavras-chave: pesquisa; Educação Física; metodologia; teoria.

\section{Para citar este artículo}

Levorattii, A. (2020). Profesores que estudian profesores. Reflexiones teórico-metodológicas sobre los procesos de investigación en y sobre la Educación Física. Lúdica Pedagógica, 1(31), 87-94. https://doi.org/10.17227/ ludica.num31-11743

\section{(c) (1) (8)}




\section{INTRODUCCIÓN}

El presente ensayo busca problematizar el proceso de investigación desarrollado en los últimos años sobre los diversos ámbitos de formación y ejercicio profesional de los profesores de Educación Física. Desde 2006, participo en proyectos de investigación cumpliendo diferentes roles. En estos contextos, estudié las representaciones sociales sobre los deportes que tenían los profesores de Educación Física y funcionarios que participaban en programas de política socioeducativa a partir de la realización de una etnografía; por otro lado, analicé los procesos de configuración de la formación docente en Educación Física en la Argentina, cumplimentando para ello trabajo en archivos institucionales, observaciones en institutos de formación y entrevistas en profundidad a directivos, docentes y estudiantes ${ }^{2}$.

En los diferentes casos, los análisis entraron en diálogo con categorías conceptuales promovidas desde diferentes disciplinas sociales, entre las que se pueden nombrar cuerpo, deporte, configuraciones, campo, habitus, política. Ahora bien, el lector involucrado en un sector de los debates de la Educación Física en las últimas décadas en la región estará familiarizado con estas discusiones. Ello se pude pensar tanto en trabajos que inscriben a la Educación Física en las ciencias sociales o en las ciencias de la educación, como así también en espacios de producción académica desde donde se promovieron análisis sobre las problemáticas y enfoques conceptuales de las ciencias sociales de y en la Educación Física ${ }^{3}$. Estas producciones ingresaron en una intersección entre categorías conceptuales y problemáticas analíticas que se fueron desarrollando en distintas disciplinas del amplio espectro de las ciencias sociales, humanas y de la edu-

2 Para la construcción de este problema de investigación se retoma la perspectiva conceptual configuracional de Norbert Elias (1996) a partir de la cual se analizan los procesos de configuración de la formación de los profesores de Educación Física, es decir que consideremos su carácter histórico, que se asumen como objeto de un devenir a lo largo del tiempo. Al estudiar las configuraciones de la formación básica de los profesores de Educación Física atendimos a los procesos históricos y las condiciones estructurales en las cuales se construyeron, pero al mismo tiempo se considera a los hombres y las mujeres que la integraron.

3 En ambos casos la producción bibliográfica es muy extensa, no citaremos autores en esta oportunidad. Posibles entradas a esta temática y autores se pueden encontrar en los números temáticos publicados en el Journal of the Latin American Sociocultural Studies of Sport (Asociación Latinoamericana de Estudios Socioculturales del Deporte-ALESDE), en el año 2018, en su volumen 9 , números 1 y 2 . cación y los debates que se venían generando en la Educación Física. Esta situación podría inducir a preguntarse si serían investigaciones de o en la educación física, cuestión que amerita un desarrollo que no me encuentro en condiciones de realizar en estas páginas.

No obstante, a partir de estas intersecciones entre las problemáticas estudiadas, las categorías conceptuales empleadas, los actores analizados, mi propia trayectoria formativa e inscripciones laborales/institucionales, me interesa ahora problematizar tres cuestiones sobre las prácticas de investigación: la primera está asociada al estudio de un espacio social donde uno participa activamente; la segunda, a las reflexividades de los profesores, los cuales son objeto de investigación y en esos procesos el empleo de categorías conceptuales compartidas por el investigador; en tercer lugar, vinculado a lo anterior, es de interés reflexionar sobre las motivaciones de los actores estudiados en nuestros trabajos y las posibles repercusiones de la obra producida en los debates de la Educación Física.

\section{ESTUDIO DEL GRUPO SOCIAL}

\section{AL QUE SE PERTENECE}

Como profesor egresado en Educación Física de la Universidad Nacional de La Plata (UNLP) y desempeñándome como docente e investigador en dicha institución, a lo largo de la investigación sobre los procesos de configuración de la formación inicial se pusieron en juego mi formación académica e inscripción universitaria de diferentes modos. Al momento de entrevistar a los funcionarios que participaron de las reformas de los planes de estudio, en el nivel nacional, provincial y en la universidad, dichas cuestiones se activaron de diferentes maneras. Los entrevistados que se desempeñaban en la UNLP me identificaban como un interlocutor radicado en una unidad de investigación particular, con vínculos personales y académicos con determinados actores. Por su parte, para los profesores no insertos académica y profesionalmente en dicha institución, esta misma inscripción adquirió otros significados, asociados con el particular modo en que ellos percibían al conjunto de actores de la UNLP en los debates disciplinares. En el proceso de investigación fui visibilizando, de forma gradual, estas cuestiones promoviendo una autorreflexión sobre mis propias categorías, internalizadas a lo largo de mi formación y socialización académica, a partir de la comparación con las perspectivas de los sujetos estudiados. 
Esto me llevó a reflexionar sobre las construcciones de los datos en el marco del trabajo de campo (Bourdieu y Wacquant, 2008; Guber, 2001). En ese sentido, durante la investigación se analizaron las afirmaciones e interpretaciones que los actores pusieron en juego en las interacciones que produjimos como parte de situaciones sociales particulares, que dan sentido a ellas, siendo necesario hacer un ejercicio reflexivo sistemático y personal sobre estas, para no caer en generalizaciones o interpretaciones apriorísticas especialmente aquellas informadas por mi propia formación como profesor en educación física, mi trayectoria e inserción académica y profesional, mis relaciones personalizadas con diferentes actores sociales que fueron objeto de estudio, y aun mi propia condición de sujeto y objeto de esta investigación- que no atiendan a las contextos de producción. Esta cuestión no se reduce solo al trabajo con las entrevistas.

También, al analizar los documentos técnicos que produjeron los funcionarios, los programas que realizaron los docentes o las encuestas que respondieron los estudiantes, se debe atender a los contextos de producción de dichos materiales y los posibles interlocutores que identificaron tanto de los fines del instrumento como así también de la figura del investigador. Es decir, un programa de una materia es un instrumento administrativo legal, con el que cuenta el docente para legitimar su hacer ante los estudiantes, pero, a su vez, puede oficiar como un instrumento de directivos o inspectores sobre el accionar docente. No contemplar ello ocasionaría no atender al contexto de producción del contenido puesto en juego en dicha instancia de interacción. En esa misma dirección, se tiende a considerar que las interpretaciones que se generaron a lo largo de las entrevistas sobre el fenómeno en cuestión hablan de una producción situacional particular (Guber, 2001; Taylor y Bogdan, 1987; Archenti et ál. 2018).

A lo largo de la investigación realicé entrevistas a profesores que se desempeñaron en las agencias estatales encargadas de definir los lineamientos curriculares para la formación de los profesores de Educación Física tanto en el Ministerio de Educación de la Nación como en la Dirección General de Cultura y Educación de la Provincia de Buenos Aires. Igualmente, entrevisté a profesores que ejercían -o lo habían hecho- en las instancias de formación docente inicial en cuatro instituciones diferentes.
Las primeras labores que cumplimenté fueron la recopilación del material documental establecido por las dos reparticiones analizadas. En ese sentido, me adentré en la búsqueda de las producciones escritas de los actores sociales que participaron en las diferentes agencias estatales, para en un tercer momento iniciar las entrevistas en profundidad. Aunque, paralelo a ello, efectué la compilación de los programas de las asignaturas en las distintas instituciones seleccionadas y la administración de las encuestas a los estudiantes.

Un elemento de las entrevistas que en su momento no advertí fue que las primeras fueron realizadas a los profesores que participaron en la configuración del documento de los contenidos básicos comunes (CBC) para la formación docente y los que participaron en la reforma del plan de estudios de la UNLP, que en muchos casos eran la misma persona. Teniendo en cuenta que mi formación inicial e inscripción institucional eran en dicha universidad, y que los entrevistados además habían sido mis profesores, muchos elementos de sus perspectivas se me presentaron en lo personal ante un velo de categorías comunes, aunque en el proceso de transcripción y análisis del material, podía ir identificando el carácter dialógico y situacional de las interpretaciones que se produjeron sobre los procesos estudiados. Recién al momento de entrevistar a los profesores que habían participado de la conformación de los documentos del Instituto Nacional de Formación Docente de 2009 y de la reforma del plan de estudios de Dirección General de Cultura y Educación de la Provincia de Buenos Aires en 1999, los cuales se posicionaban en un abordaje conceptual disímil al cual me había socializado, e ir aprendiendo su perspectiva sobre los procesos sociales estudiados, pude relativizar muchas de las categorías que organizaban la formación. Ello me ubicó en una relación distinta con los posicionamientos que afronté inicialmente.

En ese punto, como afirma Rosana Guber, citando a Marisa Peirano "que el conocimiento se revela no 'al' investigar sino 'en' el investigador, debiendo comparecer en el campo, debiendo reaprenderse y reaprender el mundo desde otra perspectiva" (Guber, 2001, p. 54). Puedo graficar esto a partir de un criterio de clasificación que tenía implícito en mi perspectiva, el cual estaba vinculado al hecho que la educación física tenía contenidos que son saberes culturales, es decir, 
son prácticas de la cultura. Con el devenir de la investigación y aprender más sobre este campo empírico desde un enfoque etnográfico, sin embargo, relativicé y comprendí mis propios criterios de percepción disciplinar. Además, esto muestra que la comparación es un elemento central para la producción del análisis, volveremos más adelante sobre ello (Balvi, 2017; Barth, 2000).

El ejercicio comparativo fue una herramienta que permitió salirse de la posición disciplinar, y así se evidenciaron problemáticas a ser estudiadas tanto al identificar las particulares de los casos como los puntos recurrentes; al mismo tiempo, fue posible visibilizar construcciones analíticas que teníamos naturalizadas como actor social del grupo y era necesario comprender para evitar los etnocentrismos o promover una investigación que reprodujera dogmáticamente una serie de principios apriorísticos.

\section{LA REFLEXIVIDAD DE LOS SUJETOS ESTUDIADOS Y EL "EFECTO DE TEORÍA"}

Como se expuso en el apartado anterior, los profesores entrevistados, en dicha situación social, pusieron en consideración interpretaciones sobre los fenómenos estudiados, que no pueden ser desancladas del contexto de interacción en el cual fueron producidos. Es decir, como investigador, solo se puede acceder a los ejercicios reflexivos que realizan los actores estudiados, lo cual, según Rosana Guber, constituye el objeto de conocimiento. Ampliando la postura de la autora, es pertinente someter a continuo análisis las reflexividades que están en el trabajo de campo:

la reflexividad del investigador en tanto que miembro de una sociedad o cultura; la reflexividad del investigador en tanto que investigador, con su perspectiva teórica, sus interlocutores académicos, sus habitus disciplinares y su epistemocentrismos y la reflexividad de la población en estudio. (2001, p. 49)

Ante esto, expresa: "La reflexividad de la población opera en su vida cotidiana y es, en definitiva, el objeto de conocimiento del investigador. Pero esta carga con dos reflexividades alternativa y conjuntamente" (2001, p. 49). La autora, recuperando los abordajes de los etnometodológos afirma:

un enunciado transmite cierta información, creando además el contexto en el cual esa información puede aparecer y tener sentido. De este modo, los sujetos producen la racionalidad de sus acciones y transforman a la vida social en una realidad coherente y comprensible. (Guber, 2001, p. 46)

$\mathrm{Al}$ respecto, se considera central analizar los ejercicios reflexivos de los actores estudiados, a raíz que se debe atender a ello en los procesos de producción de los datos.

En los enunciados de los entrevistados se observó un juicio reflexivo y crítico de los procesos sociales en los cuales ellos mismos habían participado, a través de la mención a categorías de las ciencias sociales para justificar y legitimar su accionar. En esa dirección, en los pasajes a continuación se advirtió cómo, de modo recurrente, determinados actores emplearon la denominación campo, recuperando a Pierre Bourdieu, autor de renombre en la sociología, para explicar y fundamentar su accionar. Es decir, en su construcción argumentativa, dichas categorías les permitieron posicionarse y analizar crítica y reflexivamente los procesos sociales en los cuales ellos mismos intervinieron.

Un profesor que participó de los debates curriculares planteó en la entrevista que:

en realidad, era una cosa de armar un campo, pero no porque yo cuestionara que ese campo fuera la enseñanza, digamos. [...]

Bueno, y así sucesivamente, entonces digo, a mí me interesa sostener, y estoy empeñado en armar ese campo, un campo en el cual podamos discutir, como quería Bourdieu, con las armas de la ciencia, es decir con argumentos lógicos, con argumentos $a h$ hoc y no ad hominem, no este. (Docente A, octubre de 2015). (Resaltado por fuera del original)

Otro entrevistado, que se encontraba dirigiendo la carrera de Educación Física al momento de modificarse los planes de estudios expresó: “[...] en mi gestión al frente del departamento fue una gestión sobre la base del concepto de la hegemonía de una idea en un campo" (Docente B, octubre de 2015) (resaltado por fuera del original).

Una funcionaria, que participó de la producción de los lineamientos nacionales para la reforma curricular, afirmó: "Es como, ir buscando argumentaciones de teóricos, que vienen a fortalecer el campo" (Docente C, septiembre de 2015) (resaltado por fuera del original). 
Los tres entrevistados emplearon la misma categoría campo para significar sus acciones. Este hecho llamó la atención, pues señaló una alerta sobre el lugar de la teoría social en las interpretaciones que los propios actores sociales hacen de sus propias prácticas. $\mathrm{Al}$ respecto, lo primero que se identificó fue un principio como efecto de teoría; es decir, cuando los actores presentaban los procesos en que formaron parte, empleaban en sus narrativas términos y conceptos de la teoría social para explicar, reflexionar y fundamentar su accionar. Esta cuestión me ubicó frente a los ejercicios reflexivos y analíticos de los actores sobre los procesos en los cuales ellos participaban, utilizando determinadas categorías analíticas que los inscribían en diálogos con las ciencias sociales y de la educación. Ante ello, en este trabajo no se busca constatar si lo que los actores planteaban era o no un campo en los términos de un determinado autor; en cambio, se procura comprender e interpretar por qué hacían referencias a esos conceptos en las producciones de los discursos sobre dichos procesos sociales.

En este estudio tuvo un lugar estructurador la teoría de Luc Bolstanski (2014), con su sociología de la crítica, la cual se enfoca en el estudio, los ejercicios de análisis y de crítica que realizan los sujetos sociales estudiados. Esto llevó a que la capacidad de crítica no se reserve al investigador, esta también es propiedad de la población estudiada, lo que hay que analizar son las competencias que tienen los diferentes actores y las disputas sobre la producción de sentidos en la cual se inscribieron. En el siguiente apartado interesa reflexionar sobre el lugar asignado a la investigación por los actores estudiados.

\section{LOS RESULTADOS DE LA INVESTIGACIÓN COMO ESPACIO DE DISPUTA}

En el desarrollo de las entrevistas, los diferentes actores sociales desglosaron sus argumentaciones en relación con sus interpretaciones sobre los procesos que aquí se estudian. Ahora bien, ¿a quién estaban destinadas estas presentaciones? Claramente a otro profesor de educación física que clasificaban y posicionaban, como vimos antes, en la disputa disciplinar. Esto llevaba a que, a partir del problema de investigación que se les planteaba - sobre el análisis de los procesos de configuración de la formación de los profesores en la Provincia de Buenos Aires-, ellos interpretaban y procuraban direccionar la problemática hacia sus inquietudes como nativos. En el siguiente fragmento de entrevista se advierte ese direccionamiento:

lo que pasa es que la formación recupera todos estos avances, esto que yo te estoy nombrando de formulaciones previas son antecedentes para tu estudio. Como se edifica esta concepción, bueno se edifica mucho en el año 86, diseño de la Ciudad [de Buenos Aires]. La Educación Física organizada en estos ejes, un eje que tiene, viste con distintos nombres apunta a eso. En la Ciudad, subsiste esta mirada de los tres ejes, eh, aunque bueno en el diseño actual de la secundaria esos ejes están bien articulados con la práctica cultural. Como la historia de los procesos son muchas veces pendulares, de mirar solamente al movimiento, bueno, se pasa como una centralidad, a veces excesiva, en el sujeto, y se pierde de vista lo sociocultural, la cultura corporal de la que habla Valter Bracht. (Docente C, septiembre de 2015). (Resaltado por fuera del original)

Esta referencia a las discusiones curriculares como antecedentes de este estudio, en primer lugar llamó la atención, pero no fue comprendido hasta mucho tiempo después, cuando al analizar el conjunto de las entrevistas donde lo que advertía era una búsqueda por parte de los profesores de posicionarme en la discusión disciplinar y en las disputas que ellos identificaban en la perspectiva nativa. Además, los funcionarios, al presentar sus perspectivas, hacían referencia a sus tesis de doctorado y maestría, finalizadas o en curso, a las cuales recurrían para fundamentar sus dichos, autoposicionándose como legítimos analistas del fenómeno y en una relación de par conmigo.

A partir de ello, dos cuestiones se transforman en centrales. La primera está asociada al hecho de no caer en etnocentrismos al momento de analizar las categorías de los actores, por ello se retoma el planteamiento de Faye Ginsburg (2004). que propone evidenciar y diferenciar nítidamente las categorías de los actores y las de investigador, con el fin de no imponer a los primeros - de un modo etnocéntricolas concepciones que sustento como investigador, al tiempo que me propongo remarcar el carácter dialógico de la producción del conocimiento etnográfico, pues la producción cultural es siempre relacional. La 
segunda cuestión está relacionada con el riesgo de transformarme en autor/analista que legitime una determinada propuesta disciplinar, y que el abordaje presente sesgos asociados a las concepciones y prácticas acerca de la Educación Física en las cuales me posiciono, reproduciendo una perspectiva nativa. Es decir, el peligro del trabajo es argumentar sobre un determinado posicionamiento conceptual de la disciplina, o asumir que una perspectiva de los actores como la interpretación autorizada sobre el fenómeno (Gil, 2007). Esto está dado por el hecho de compartir con los actores categorías conceptuales, que ellos utilizan para interpretar los fenómenos y dar sentido a sus procesos, y en mi caso forman parte de mis herramientas analíticas. Por esta razón, por momentos las interpretaciones nativas - con conceptos de las ciencias sociales incluidas - se aproximan íntimamente a las comprensiones del fenómeno analizado.

Así, recuperando lo planteando, se considera que los actores estudiados producen interpretaciones sobre los fenómenos en cuestión. Ahora bien, en este caso, dichos abordajes forman parte de la categorías nativas o sociales (Rockwell, 1987). Entendiendo que los distintos actores estudiados tienen posicionamientos situados e informados desigualmente, la tarea del investigador es producir una comprensión densa de dichos procesos, utilizando para ello categorías teóricas (Rockwell, 1987). Esta labor además tiene por fin discutir los abordajes conceptuales. Lo planteado busca diferenciar y visibilizar los niveles de análisis pues, como investigadores, podemos terminar legitimando o invisibilizando determinadas perspectivas. En ese sentido, al reproducir acríticamente es posible terminar reproduciendo determinadas narrativas y nuestra labor contribuyendo a la legalización e instauración de memorias singulares del grupo.

\section{A MODO DE CIERRE}

En este breve ensayo se buscó poner el foco en las implicancias de estudiar el mismo espacio social en el cual uno se desenvuelve profesionalmente. Esta cuestión es recurrente en la educación física al inscribirse el investigador también como actor social socializado en la disciplina. En estos casos, la labor implica la realización de diversos ejercicios de análisis y de reflexión, los cuales entendemos que hacen al proceso de cons- trucción de los datos. Teniendo en cuenta lo planteado hasta acá y recuperando a Alexandre Fernández Vaz (2007). quien sugiere que lo relevante de la pesquisa es que cambie la mirada del investigador, entendemos que ello es central para poder conocer más los procesos sociales en los cuales nos inscribimos y como consecuencia a la Educación Física.

Las reflexiones metodológicas se encuentran inscritas y en permanente funcionamiento dentro de una perspectiva teórica que se asume para comprender al fenómeno.

Por último, estas páginas buscaron visibilizar y reflexionar sobre los diferentes procesos transitados durante el trabajo de campo. Se considera que invisibilizar tales instancias negaría las tramas en las cuales nos inscribimos cuando investigamos y a la modalidad de construcción de conocimiento sobre la disciplina. Por ello que no se puede olvidar ni desentender al hecho de que las producciones resultantes de estos procesos hacen a la construcción de la Educación Física.

\section{REFERENCIAS}

Balvi, F. (2017). La comparación en antropología social: problemas y perspectivas. Antropofagia.

Barth, F. (2000). o guru, o iniciador e outras variações antropológicas. Contracapa.

Boltanski, L. (2014). De la crítica. Compendio de sociología de la emancipación. Akal.

Bourdieu, P. y Wacquant, L. (2008). Una invitación a la sociología reflexiva. Siglo XxI.

Elias, N. (1996). La sociedad cortesana. Fondo de Cultura Económica.

Fernandez Vaz, A. (2007). Anotaciones sobre la relación entre subjetividad y objetividad en el proceso de investigación: ocho ideas sobre la elaboración de proyectos. Efdeportes.com, 12(108).

Gil, G. (2007). Hinchas en tránsito: violencia, memoria e identidad en una hinchada de un club del interior. Editorial de la Universidad Nacional de Mar del Plata.

Ginsburg, F. (2004). Cuando los nativos son nuestros vecinos. En M. Boivin, A. Rosato y V. Arribas (eds.), Constructores de otredad. Una introducción a la antropología social y cultural (pp. 186-193). Editorial Antropofagia. 
Guber, R. (2001). La etnografía. Método, campo y reflexividad. Norma.

Rockwell, E. (1987). Reflexiones sobre el proceso etnográfico. Departamentos de Investigaciones Educativas (DIE).
Visacovsky, S. (2005). El temor a escribir sobre historia sagradas. Memoria social, moralidad política y audiencias nativas en la Argentina. En: F. Sabina y G. Soprano (comps.), Cultura y política en etnografías sobre la Argentina (pp. 271-313). Universidad Nacional de Quilmes Editorial. 